\title{
Generalization and Modification of Hardy-Littlewood Maximal Functions
}

\section{${ }^{* 1}$ ADEWINBI, HS; ${ }^{2}$ PETER, OJ}

\author{
${ }^{I}$ Department of Mathematics, University of Ibadan, Ibadan, Oyo State, Nigeria. \\ ${ }^{2}$ Department of Mathematics, University of Ilorin, llorin, Kwara State, Nigeria. \\ Corresponding Author: ayedunseun@gmail.com 07033221618
}

\begin{abstract}
The purpose of this paper is to provide the different types of Hardy-Littlewood Maximal Functions, the relationship between them and the corresponding extension of $\mathbb{R}^{n}$ of the Hardy-Littlewood maximal function. We also give the generalization and the modification of Hardy-Littlewood maximal function.
\end{abstract}

DOI: https://dx.doi.org/10.4314/jasem.v22i3.18

Copyright: Copyright $@ 2018$ Adewinbi and Peter. This is an open access article distributed under the Creative Commons Attribution License (CCL), which permits unrestricted use, distribution, and reproduction in any medium, provided the original work is properly cited

Dates: Received: 02 February 2018; Revised: 28 February: 2018; Accepted: 20 March 2018

Keywords: Maximal function, Hardy-Littlewood Maximal Functions, Borel measure, Fourier coefficient.

Maximal functions arise very natural in analysis, for proving theorems about the existence almost everywhere of limits, for controlling pointwise important objects such as the Poisson Integrals or for controlling, not pointwise but at least in average, other basic operators such as singular integral operators. The model example of existence almost everywhere of limits is the Lebesgue differentiation theorem:

$$
f(x)=\lim _{r \rightarrow 0} \frac{1}{|B(x, r)|} \int_{B(x, r)} f(y) d y
$$

This property is intimately related to the study of certain properties of the Hardy-Littlewood maximal function. It is a classical mean operator, and it is frequently used to majorize other important operators in harmonic analysis. There are other almost everywhere convergence problems in mathematics: Fourier series, Dirichlet problem, the heat equation, the Schrodinger equation, conjugate functions, Hilbert transforms, ergodic theory, harmonic functions, singular integrals etc. All of them have the same pattern; we are interested in maximal operator. The key property to understand the Hardy-Littlewood maximal operator is the so called "weak type" estimate or property of M. Several mathematicians have worked on Hardy-Littlewood maximal functions. For example (Mingquan, 2016) proof that for $1<p<\infty$, the $L^{p}$ norm of the truncated centered Hardy-Littlewood maximal operator $M_{\alpha}^{c}$ equals the norm of the centered Hardy-Littlewood maximal operator for all $0<\alpha<\infty$. (Martin-Reyes, 1993) gives simple proof of the characterization of the weights for which the one-sided Hardy-Littlewood maximal functions apply $L^{p}(W)$ into $L^{p}(W)$, where $\mathrm{W}$ is a nonnegative measurable function. We will like to extend the existing work in (Martin-Reyes, 1993) by looking at the generalization and modification of Hardy-Littlewood maximal functions.

Hardy-Littlewood Maximal Function

Defintion 1.1: Given $f \in L_{l o c}^{1}(\mathbb{R})$, we define

$$
M f(x)=\sup _{x \in B} \frac{1}{|B|} \int_{B}|f(y)| d y
$$

where $\mathrm{B}$ is an integral containing $\mathrm{x}$, and $|B|$ is the Lebesgue measure of $\mathrm{B}$. The function $M f(x)$ is called the maximal function of Hardy-Littlewood and the operator

$$
M: f \mapsto M f
$$

It is called Hardy-Littlewood's maximal operator. M is not linear but sub-linear in the sense that

$$
\begin{aligned}
& \quad M(f+g) \leq M f+M g, M(\alpha f)=|\alpha| M f \\
& \quad \text { Proof } \\
& M f(x)=\sup _{x \in B} \frac{1}{|B|} \int_{B}|f(y)| d y \\
& M(f+g)(x)=\sup _{x \in B} \frac{1}{|B|} \int_{B}|f(y)+g(y)| d y \\
& \leq \sup _{x \in B} \frac{1}{|B|} \int_{B}(|f(y)|+|g(y)|) d y \\
& =\sup _{x \in B} \frac{1}{|B|} \int_{B}|f(y)| d y+\sup _{x \in B} \frac{1}{|B|} \int_{B}|g(y)| d y \\
& =M f(x)+M g(x) \\
& =(M f+M g)(x) \\
& \therefore M(f+g) \leq M f+M g
\end{aligned}
$$


Generalization and Modification of Hardy-Littlewood.....

Also

$$
\begin{aligned}
& M(\alpha f)(x)=\sup _{x \in B} \frac{1}{|B|} \int_{B}|\alpha f(y)| d y \\
& =\sup _{x \in B} \frac{1}{|B|} \int_{B}|\alpha||f(y)| d y \\
& =|\alpha| \sup _{x \in B} \frac{1}{|B|} \int_{B}|f(y)| d y \\
& =|\alpha| M f(x) \\
& \therefore M(\alpha f)=|\alpha| M f \\
& \text { Combining (2) and (3) the result follows } \\
& \text { immediately. }
\end{aligned}
$$

Definition 1.2: The one-sided maximal functions $M^{+} f$ and $M^{-} f$ of a function $f \in L_{l o c}^{1}(\mathbb{R})$ is given by (Sawyer, 1986) as

$$
\begin{aligned}
& M^{+} f=\sup _{h>0} \frac{1}{h} \int_{x}^{x+h}|f| \\
& M^{-} f=\sup _{h>0} \frac{1}{h} \int_{x-h}^{x}|f|
\end{aligned}
$$

The operators $M^{+} f(x)$ and $M^{-} f(x)$ are interesting because they control some one-sided operators such as singular integrals with kernels supported in $(-\infty, 0)$ or $(0, \infty)$.

The results of the operators $M_{g}^{+}$and $M_{g}^{-}$are defined as

$$
\begin{aligned}
M_{g}^{+} f(x) & =\sup _{h>0} \frac{\int_{x}^{x+h}|f| g}{\int_{x}^{x+h} g} \\
M_{g}^{-} f(x) & =\sup _{h>0} \frac{\int_{x-h}^{x}|f| g}{\int_{x-h}^{x} g}
\end{aligned}
$$

Where $\mathrm{g}$ is a positive locally integrable function.

Lemma 1.1: The function $M f$ is lowersemicontinuous, hence measurable.

\section{Proof}

Let $M\left(x_{0}\right)>\beta$. Then there is a ball $\mathrm{B}$ containing $\mathrm{x}$ such that

$$
\frac{1}{|B|} \int_{B}|f(y)| d y>\beta
$$

Then $M f(x)>\beta$ for every $x \in B$.

Definition 1.3: The classsical Hardy-Littlewood maximal function is given by

$$
M^{\prime} f(x)=\sup _{r>0} \frac{1}{|B(x, r)|} \int_{B(x, r)}|f(y)| d y
$$

i.e limited to the averages of $|f|$ over balls centered at $\mathrm{x}$.

clearly, $M^{\prime} f(x) \leq M f(x)$

$M^{\prime} f$ is not necessarily lower-semicontinuous. The measurability of $M^{\prime} f$ follows from the fact that the map

$$
F(x, r)=\frac{1}{|B(x, r)|} \int_{B(x, r)}|f(y)| d y
$$

is continuous in $r$, so that the sup in (4) can be limited to $r \in \mathbb{Q}$.

Definition 1.4: The centered Hardy-Littlewood maximal function is given by

$$
M^{c} f(x)=\sup _{r>0} \frac{1}{|B(x, r)|} \int_{B(x, r)}|f(y)| d y
$$

and the uncentered Hardy-Littlewood maximal function is

It is clear that

$$
M f(x)=\sup _{x \in B} \frac{1}{|B|} \int_{B}|f(y)| d y
$$

$$
2^{n} M^{c} f(x) \geq M f(x) \geq M^{c} f(x)
$$

holds for all $x \in \mathbb{R}^{n}$. Both $M$ and $M^{c}$ are sublinear operators. It is very difficult to calculate the exact norm of $M$ and $M^{c}$. The basic real-variable construct was introduced by (Hardy and Littlewood, 1930) for $n=1$ and (Wiener, 1939) for $n \geq 2$.

Definition 1.5: Trucated Centered Hardy-Littewood maximal operator is given by

$$
M_{\alpha}^{c} f(x)=\sup _{0<r<\alpha} \frac{1}{|B(x, r)|} \int_{B(x, r)}|f(y)| d y
$$

and the Trucated Uncentered Hardy-Littlewood maximal operator

$$
\begin{aligned}
& M_{\alpha} f(x) \\
& =\sup _{0<r<\alpha,|y-x|<r} \frac{1}{|B(y, r)|} \int_{B(y, r)}|f(t)| d t
\end{aligned}
$$

For $x \in \mathbb{R}^{n}$ and some real positive number $\alpha$.

We can deduce from (5), (6) and (7) that

$$
\begin{aligned}
& M^{c} f(x) \geq M_{\beta}^{c} f(x) \geq M_{\alpha}^{c} f(x) \\
& \text { and } \\
& M f(x) \geq M_{\beta} f(x) \geq M_{\alpha} f(x) \\
& \forall x \in \mathbb{R}^{n} \text {, provided } \alpha \leq \beta \\
& \text { It follows from (8) and (9), as the sublinear operators } \\
& \left\|M^{c}\right\|_{L^{p}\left(\mathbb{R}^{n}\right) \rightarrow L^{p}}\left(\mathbb{R}^{n}\right) \geq\left\|M_{\beta}^{c}\right\|_{L^{p}}\left(\mathbb{R}^{n}\right) \rightarrow L^{p}\left(\mathbb{R}^{n}\right) \\
& \qquad \\
& \left.\quad \geq\left\|M_{\alpha}^{c}\right\|_{L^{p}} \mathbb{R}^{n}\right) \rightarrow L^{p}\left(\mathbb{R}^{n}\right)
\end{aligned}
$$

and 
Generalization and Modification of Hardy-Littlewood.....

$$
\begin{array}{r}
\left.\|M\|_{L^{p}} \mathbb{R}^{n}\right) \rightarrow L^{\left.p_{(\mathbb{R}}^{n}\right)} \\
\geq\|\| M_{\beta} \|_{L^{p}}{ }_{\left(\mathbb{R}^{n}\right) \rightarrow L^{p}}{ }_{\left(\mathbb{R}^{n}\right)} \\
\geq\left\|M_{\alpha}\right\|_{L^{p}\left(\mathbb{R}^{n}\right) \rightarrow L^{p}\left(\mathbb{R}^{n}\right)}
\end{array}
$$

If $\alpha \leq \beta$, for $1<p \leq \infty$.

This shows that for $1<p \leq \infty$, the $L^{p}\left(\mathbb{R}^{n}\right)$ norm of the centered Hardy-Littlewood maximal operator is greater or equals to trucated centered HardyLittlewood maximal operator. Also, for $1<p \leq \infty$, the $L^{p}$ norm of the uncentered Hardy-Littlewood maximal operator is greater or equals to trucated uncentered Hardy-Littlewood maximal operator.

Relationship Between Hardy-Littewood Maximal Operators

Theorem 2.1: Let $M_{\alpha}^{c}$ be defined by (6) and $\alpha>0$. Then

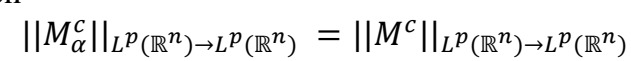

That is, the norm of Trucated Centered HardyLittlewood maximal operator and the Trucated Uncentered Hardy-Littlehood maximal operator are exactly the same on a Lebsegue measure[6].

Theorem 2.2: Let $M_{\alpha}^{c}$ be defined by (6) and $\alpha>0$. Then

$$
\left\|M_{\alpha}^{c}\right\|_{L^{1}\left(\mathbb{R}^{n}\right) \rightarrow L^{1, \infty}\left(\mathbb{R}^{n}\right)}=\left\|M^{c}\right\|_{L^{1}\left(\mathbb{R}^{n}\right) \rightarrow L^{1, \infty}\left(\mathbb{R}^{n}\right)}
$$

Theorem 2.3: Let $M_{\alpha}$ be defined by (7) and $\alpha>0$. Then

$$
\left\|M_{\alpha}\right\|_{L^{1}\left(\mathbb{R}^{n}\right) \rightarrow L^{1, \infty}\left(\mathbb{R}^{n}\right)}=\|M\|_{L^{1}\left(\mathbb{R}^{n}\right) \rightarrow L^{1, \infty}\left(\mathbb{R}^{n}\right)}
$$

Theorem 2.4: Let $M_{\alpha}$ be defined by (7) and $\alpha>0$. Then

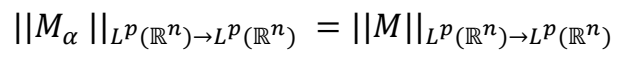

Lemma 2.1: Suppose that $\mu$ is a positive measure on a $\delta$-algebra $\mathbb{M}$. If $A_{1} \subset A_{2} \subset A_{3} \ldots A_{n} \in \mathbb{M}$, and $A=\bigcup_{n=1}^{\infty} A_{n}$, then

$$
\lim _{n \rightarrow \infty} \mu\left(A_{n}\right)=\mu(A)
$$

Lemma 2.2: Suppose that the operators $M^{c}$ and $M_{\alpha}^{c}$ are defined as in (8) and (10). The equality

$$
d M^{c} f(\lambda)=\lim _{\alpha \rightarrow \infty} d M_{\alpha}^{c} f(\lambda)
$$

Holds for all $f \in L^{p}\left(\mathbb{R}^{n}\right)$ and $\lambda>0$.

\section{Proof}

For a fixed $x \in \mathbb{R}^{n}$, by the definition of $M^{c}$ in (8), associate to each $\varepsilon$ a ball $B\left(x, r_{\varepsilon}\right)$ which satisfies

$\frac{1}{\left|B\left(x, r_{\varepsilon}\right)\right|} \int_{B\left(x, r_{\varepsilon}\right)}|f(y)| d y>M^{c} f(x)-\varepsilon$
Now taking $\alpha>r_{\varepsilon}$, it follows from the definition of $M_{\alpha}^{c}$ that

$$
M_{\alpha}^{c} f(x) \geq \frac{1}{\left|B\left(x, r_{\varepsilon}\right)\right|} \int_{B\left(x, r_{\varepsilon}\right)}|f(y)| d y>M^{c} f(x)-\varepsilon
$$

Note that $M_{\alpha}^{c} f(x)$ increases as $\alpha \rightarrow \infty$. Thus we have

$$
\lim _{\alpha \rightarrow \infty} M_{\alpha}^{c} \geq M f
$$

Clearly, we have

$$
M_{\alpha}^{c} f \leq M f
$$

Combining (12) and (13), we have

$$
\lim _{\alpha \rightarrow \infty} M_{\alpha}^{c} f=M^{c} f
$$

Then

$$
\lim _{n \rightarrow \infty} M_{n}^{c} f=M^{c} f
$$

We set

and

$$
A_{n}=\left\{x \in \mathbb{R}^{n}: M_{n}^{c} f(x)>\lambda\right\}
$$

$$
A=\left\{x \in \mathbb{R}^{n}: M^{c} f(x)>\lambda\right\}
$$

We have $A_{n} \subset A_{n+1}$ for $n=1,2, \ldots$, and

$A=\mathrm{U}_{n=1}^{\infty} A_{n}$. It follows from Lemma 2.1 and the definition of the distribution function that

$$
\begin{aligned}
& \begin{aligned}
d M^{c} f(\lambda)=|A| & =\lim _{n \rightarrow \infty}\left|A_{n}\right| \\
& =\lim _{n \rightarrow \infty} d M_{n}^{c} f(\lambda)=\lim _{\alpha \rightarrow \infty} d M_{\alpha}^{c} f(\lambda)
\end{aligned} \\
& \text { This is our desired result. }
\end{aligned}
$$

Generalization Of One-Sided Maximal Function

The natural generalization of $M^{+}$in $\mathbb{R}^{n}$ is the following: given $x=\left(x_{1}, x_{2}, \ldots x_{n}\right)$ we have

$$
M^{+\cdots+} f(x)=\sup _{h>0} \frac{1}{h^{n}} \int_{Q_{x}(h)}|f(y)| d y
$$

Where

$$
Q_{x}(h)=\left[x_{1}, x_{1}+h\right) \times\left[x_{2}, x_{2}+h\right) \times \ldots \times\left[x_{n}, x_{n}+h\right)
$$

In $\mathbb{R}$ we have two one-sided operators. In $\mathbb{R}^{n}$ we obviously have $2^{n}$ one-sided operators that we do no write explicitly.

Given $x=\left(x_{1}, x_{2}, \ldots x_{n}\right) \in \mathbb{R}^{n}$, let us assume 
Generalization and Modification of Hardy-Littlewood.....

$$
N^{+\cdots+} f(x)=\sup _{h>0} \frac{1}{h^{n}} \int_{Q_{x}^{+}(h)}|f(y)| d y
$$

Where

$$
Q_{x}^{+}(h)=\left[x_{1}+h, x_{1}+2 h\right) \times\left[x_{2}+h, x_{2}+2 h\right) \times \ldots \times\left[x_{n}+h, x_{n}+2 h\right)
$$

For $n=1, M^{+}$and $N^{+}$are equivalent.

It is clear that $N^{+\cdots+} f(x) \leq 2^{n} M^{+\cdots+} f(x)$ but there is no constant $C>0$ satisfying

$C N^{+\cdots+} f(x) \geq M^{+\cdots+} f(x)$

for $n>1$.

If $Q=\left[x_{1}, x_{1}+h\right) \times\left[x_{2}, x_{2}+h\right) \times \ldots \times\left[x_{n}, x_{n}+\right.$ $h)$ is a cube denote

$Q^{-}=\left[x_{1}, x_{1}+\frac{h}{2}\right) \times\left[x_{2}, x_{2}+\frac{h}{2}\right) \times \ldots \times\left[x_{n}, x_{n}+\frac{h}{2}\right)$ and

$Q^{+}=\left[x_{1}+\frac{h}{2}, x_{1}+h\right) \times\left[x_{2}+\frac{h}{2}, x_{2}+h\right) \times \ldots \times\left[x_{n}+\frac{h}{2}, x_{n}+h\right)$

Let $A_{x}^{+}=\left\{\right.$Qdyadic $\left.: x \in Q^{-}\right\}$

The one-sided dyadic maximal function is defined by

$$
\begin{aligned}
& M_{d}^{+\cdots+} f(x)=\sup _{Q \in A_{x}^{+}} \frac{1}{\left|Q^{+}\right|} \int_{Q^{+}}^{\cdot}|f(y)| d y \\
& M_{d} f(x)=\sup _{Q \text { dyadic: } x \in Q} \frac{1}{\left|Q^{+}\right|} \int_{Q}|f(y)| d y
\end{aligned}
$$

$M_{d}$ is the classical dyadic maximal operator.

Theorem 3.1: Let $f \in L^{1}(\mathrm{R})$. Then, for every $t>0$, the set

$$
E_{t}=\{x \in \mathrm{R}: M f(x)>t\}
$$

satisfies the following

$$
\left|E_{t}\right| \leq \frac{2}{t} \int_{E_{t}}|f(x)| d x
$$

Theorem 3.2: Let $\mathrm{f}$ measurable in $\mathrm{R}$ and $t>0$. Then

$\left|E_{t}\right| \leq \frac{4}{t} \int_{\left\{x:|f(x)|>\frac{t}{2}\right\}}|f(x)| d x$

\section{Proof}

We can assume that $f \in L^{1}$. Define

$$
f_{1}=\left\{\begin{array}{cc}
f(x) & \text { if }|f(x)|>\frac{t}{2} \\
0 & \text { otherwise }
\end{array}\right.
$$

Then, $f=f_{1}+f_{2}$, with $\left|f_{2}\right| \leq \frac{t}{2}$ and $f_{i} \in L^{1}, i=1,2$ we have $M f_{2} \leq \frac{t}{2}$

and

$$
M f(x) \leq M f_{1}(x)+M f_{2}(x) \leq M f_{1}(x)+\frac{t}{2}
$$

Then

$$
E_{t} \subset\left\{x: M f_{1}(x)>\frac{t}{2}\right\}
$$

Now apply Theorem 3.1 to $f_{1}$ to obtain

$$
\begin{aligned}
\left|E_{t}\right| & \leq\left|\left\{x: M f_{1}(x)>\frac{t}{2}\right\}\right| \\
& \leq \frac{4}{t} \int_{R}\left|f_{1}(x)\right| d x \\
& =\frac{4}{t} \int_{\left\{x: f(x)>\frac{t}{2}\right\}}\left|f_{1}(x)\right| d x \\
& =\frac{4}{t} \int_{\left.\{x: f(x))>\frac{t}{2}\right\}}|f(x)| d x
\end{aligned}
$$

Theorem 3.3: Given $1<p<\infty$ there exist a constant $C_{p}$ such that for every $f \in L^{p}$

$\underline{\text { Proof }}$

$$
\|M f\|_{L^{p}} \leq C_{p}\|f\|_{L^{p}}
$$

We will use distribution function, Theorem 3.1, 3.2 to obtain

$$
\begin{aligned}
\int_{\mathbb{R}}\left(M f(x)^{p} d x\right. & =p \int_{0}^{\infty} t^{p-1}|\{x: M f(x)>t\}| d t \\
& \leq p \int_{0}^{\infty} t^{p-1} \frac{4}{t} \int_{\left\{x:|f(x)| \frac{t}{2}\right\}}|f(x)| d x d t \\
& =4 p \int_{0}^{\infty} t^{p-1} \int_{\left\{x:|f(x)| \frac{t}{2}\right\}}|f(x)| d x d t \\
& =4 p \int_{0}^{\infty} \int_{0}^{2|f(x)|} t^{p-2}|f(x)| d x d t \\
& =4 p \frac{2^{p-1}}{p-1} \int_{R}|f(x)|^{p} d x
\end{aligned}
$$

\section{Modified Hardy-Littlewood Maximal Function}

Defintion 4.1: Let $\mathrm{f}$ be a nonnegative extended realvalued Lebesgue measurable function on $\mathbb{R}$ and $\lambda$ be Lebesgue for $\mathbb{R}$. Then

$$
\begin{aligned}
& M_{r} f(x)=\sup \left\{\frac{1}{\lambda(I)} \int_{I} f d \lambda: I=[x, u], x<u<\infty\right\} \\
& M_{l} f(x)=\sup \left\{\frac{1}{\lambda(I)} \int_{I}^{f} f d \lambda: I=[u, x],-\infty<\mathrm{u}<\mathrm{x}\right\}
\end{aligned}
$$


$M f(x)=\sup \left\{\frac{1}{\lambda(I)}: I\right.$ is a closed interval containing $\left.\mathrm{x}\right\}$ $M_{r} f(x), M_{l} f(x)$ and $M f(x)$ are three diferent maximal averages of the functions $f$. All of the maximal theorems are inequalities giving bounds for the integral of one of the maximal functions composed with a monotonic function. The mapping $M_{r}, M_{l}$ and $M$ carry certain function spaces to others.

Theorem 4.1: $M f=\operatorname{Max}\left(M_{r} f, M_{l} f\right)$

See the proof in (Keith, 1965).

Definition 4.2: Let $\mathrm{f}$ be a locally integrable function on a metric measure space $(X, \mu)$. Then the $\mathrm{k}$ times modified centered Hardy-Littlewood maximal function $M_{k} f$ of $\mathrm{f}$ is defined as follows

$M_{k} f=\sup _{r>0} \frac{1}{\mu(B(x, k r))} \int_{B(x, r)}|f(y)| d \mu(y)$

We call the operator $M_{k}$ the $\mathrm{k}$ times modified centered Hardy-Littlewood maximal operator. The $\mathrm{k}$ times modified uncentered Hardy-Littlewood maximal function $M_{k} f$ of $\mathrm{f}$ is defined as follows

$M_{k, u c} f(x)=\sup _{x \in B(y, r)} \frac{1}{\mu(B(x, k r))} \int_{B(x, r)}|f(z)| d \mu(z)$

Clearly, the pointwise ineqiualities

and

$$
M_{k} f \leq M_{k^{\prime}}\left(k^{\prime} \leq k\right)
$$

$$
M_{k, u c} f \leq M_{k^{\prime}, u c}\left(k^{\prime} \leq k\right)
$$

holds for any locally integrable function $\mathrm{f}$ on $(X, \mu)$. $M_{k, u c} f(x)$ is lower semicontinuous for any locally integrable function $\mathrm{f}$.

Defintion 4.3: The n-dimensional maximal operator $\mathrm{M}$ is said to satisfy a weak type $(1,1)$ inequality if there exists a constant $\mathrm{c}$ such that for every $f \in$ $L^{1}\left(\mathbb{R}^{n}\right)$ and every $\alpha>0$ we have

$$
\alpha|\{M f>\alpha\}| \leq c|| f \|_{1}
$$

The corresponding extension to $\mathbb{R}^{n}$ of the HardyLittlewood maximal function is given by

$$
M f(x)=\sup _{r>0} \frac{1}{|B(x, r)|} \int_{B(x, r)}|f(y)| d y
$$

As in the one dimensional case this definition corresponds to the centered maximal function and for the uncentered we require the basis simply to contain the point. Note that the uncentered is contained in a centered ball with double radius. More generally, one can start with a fixed set B containing the origin and define a maximal function using all the family of sets obtained using dilations and translations of B:

$$
M_{B} f(x)=\sup _{r>0} \frac{1}{|r B|} \int_{r B}|f(x+y)| d y
$$

If there are two balls centered at the origin with radii $r_{1}$ and $r_{2}$ such that $B\left(0, r_{1}\right) \subset B \subset B\left(0, r_{2}\right)$, then $M_{B}$ is equivalent to $\mathrm{M}$ in the sense that the quotient $M f(x) / M_{B} f(x)$ is bounded above and below by constant depending only on $r_{1}, r_{2}$ and the dimension, and not on $\mathrm{f}$ or $\mathrm{x}$. In particular, this is true when $\mathrm{B}$ is the ball defined by an $l^{p}$-norm in $\mathbb{R}^{n}$.

Conclusion: In this paper, the research shows the centered Hardy-Littlewood maximal function and uncentered Hardy-Littlewood maximal funtion together with their $\mathrm{k}$ times modification and the generalization of one-sided maximal functions.

\section{REFERENCES}

Fulvio, R (2004). Hardy Spaces In One Complex Variable. Interscience Publishers, lnc., New York

Hardy, GH; Littlewood, JE(1930). A maximal theorem with function-theoretic applications, Acta Math, 54:81-116.

Keith, P (1965). Maximal Theorems of Hardy and Littlewood, University of Washington and California Institute of Technolog:648-660.

Maria, L; Martin-Reyes, FJ (2017). A note On weighted inequalities for a one-sided maximal operator in $\mathbb{R}^{n}$, Union Matematica Argentina,58,(2):253-258.

Martin-Reyes, FJ (1993). New proofs of weighted inequalities for the one-sided Hardy-Littlewood maximal functions, Americal Mathematical Society, 117, (3): 691-698.

Mingquan, W; Xudong, N; Di, W; Dunyan, Y (2016). A note on Hardy-Littlehood maximal Operators, Journal of Inequalities Applications, 21:1-13.

Sawyer, E (1986). Weighted inequalities for the onesided Hardy-Littlewood Maximal Functions, Trans. Amer.

Wiener, N (1939). The ergodic theorem. Duke Math. J. 5:1-18. 